\title{
Preliminary Data Regarding the Nontuberculous Mycobacteria Isolation from Clinical Specimens, from Human PatienTS, in Iași County
}

\author{
Ioana Alexandra RĂȚOI (ANTON) ${ }^{1 *}$, Luciana Alexandra CRIVEI ${ }^{1}$, Luanda Elena OȘLOBANU ${ }^{1}$, \\ Daniela DICULENCU ${ }^{2}$, Gheorghe SAVUȚA ${ }^{1}$ \\ ${ }^{1}$ Department of Public Health. The University of Agricultural Sciences and Veterinary Medicine Iaşi, \\ România, \\ ${ }^{2}$ Clinical Hospital of Pneumology, Iasi,România \\ * corresponding author: ioana.anton10@yahoo.com \\ Bulletin UASVM Veterinary Medicine 75(1)/2018 \\ Print ISSN 1843-5270; Electronic ISSN 1843-5378 \\ doi:10.15835/buasvmcn-vm:000118
}

\begin{abstract}
The nontuberculous mycobacteria (NTM) represents a large group ofubiquitous environmental mycobacterial species, with more than 160 members that can pose a threat for human health, especially for the immunocompromised hosts. The aim of the current study was the collection of data regarding the frequency of NTM isolation from human clinical specimens, thus providing preliminary data regarding the infection prevalence in Iași County, Romania.Between May 2015 and March 2017 epidemiological data regarding NTM isolatesin the Bacteriology Laboratory at the Clinical Hospital of Pneumology in Iasi were analyzed. A total of 63 mycobacteria strains were isolated and identified as nontuberculous (by smear microscopy, culture on solid and liquid media and immunochromatographic assay). Out of the total number of samples that were submitted in the laboratory for the diagnosis of tuberculosis, during the studied period, $0.25 \%$ of them were identified as NTM. The prevalence for 2015 was estimated at $0.18 \%$, and $0.34 \%$ for 2016 . Given the challenging diagnostic procedures and the absence of notifiable status of NTM in most European Union countries, the epidemiological situation is broadlyunknown. This emphases the need of regular recording and reporting of NTM isolation frequency for awareness of the risk of associated mycobacterial infections.
\end{abstract}

Keywords: Nontuberculous mycobacteria, NTM,Mycobacterium

\section{Introduction}

Related to the bacteria that causes human and animal tuberculosis (Mycobacterium tuberculosis complex-MTC) and leprosy ( $M$. leprae), the nontuberculous mycobacteria (NTM) represents a large heterogenic group, with over 160 species officially recognized, less than a quarter being familiar to clinicians or microbiologists (Panagiotou et al., 2014). Also referred to by other terms, such as atypical mycobacteriaor MOTT- mycobacteria other than tuberculosis, their existence was recognized shortly after the discovery of tuberculous bacillus, at the end of the 1800s (Johnson and Odell, 2014).
The mycobacterial diseases have gradually gained importance and have come to theattention of researchers, veterinarians and human doctors, reported as etiologic agent of various diseases in a wide host range, from humans to numerous animal species, such as livestock, pets or wildlife. NTM are often isolated from the environment, mainly in water, soil or vegetation. Despite the fact that NTMs are characterized by moderate pathogenicity, the incidence of infections caused by it has currently registered an increase, especially in developed countries (Rindi and Garzelli, 2016). A rise in NTM isolation rates from patients with cystic fibrosis was recorded in Europe, in the 
last years (Qvist et al., 2015). M. avium complex (MAC), M. gordonae, M. xenopi,M. intracellulare and $M$. fortuitum are amongst the most commonly isolated NTM species, with implications in human pathology (van der Werf et al., 2014; Hoefsloot, van Ingen et al., 2013). Clinical manifestations include pulmonary disease, disseminated disease, lymphatic, skin, soft tissue orbone disease. Given the fact that person to person transmission is considered extremely rare, NTM disease is not reportable in most countries (Griffith et al., 2007).

In our country, there is a significant lack of knowledge concerning the diversity and the incidence of the main NTM species involved in human and veterinary pathology. Thus, the aim of the current study was the assessment of preliminary data regarding NTM isolation from clinical specimens, in the Bacteriology Laboratory of the Clinical Hospital of Pneumology in Iasi, from June 2015 to March 2017.

\section{Materials and Methods}

The study was conducted over a period of 21 months, from June 2015 to March 2017, a total of 63 mycobacterial isolates were identified as NTM in the Bacteriology Laboratory at the Clinical Hospital of Pneumology, in Iasi. Aspects regarding primary isolation, specimen diversity, patients' age range distribution and gender were examined.

The isolation procedure starts with the digestion and the decontamination of pathological products using modified Petroff's method, with 4\% $\mathrm{NaOH}$ and $\mathrm{HCl}$, in order to remove any undesirable contaminant. The obtained sediment is inoculated into Löwenstein Jensen (LJ) solid media (the current "gold standard") and on liquid media - a Middlebrook 7H9 Broth base with a fluorescent indicator, used by the automated system present in the laboratory: BACTEC MGIT 960 (Becton Dickinson and Company), in this way shortening the incubation time. In general, the incubation period is 2 month, at a temperature of $37^{\circ} \mathrm{C}$. Each positive culture is then morphologically examined. Ziehl-Neelsen and fluorescent AuramineRhodamine stains are used for the microscopic examination of each clinical specimen.

NTM differentiation from the members of the Mycobacterium tuberculosis Complex was accomplished by using a simple and rapid immunochromatographic test - SD Bioline TB Ag MPT64 RAPID® (Standard Diagnostics, Inc., Yongin, South Korea), based on the identification of the MPT64 protein, secreted in the growth

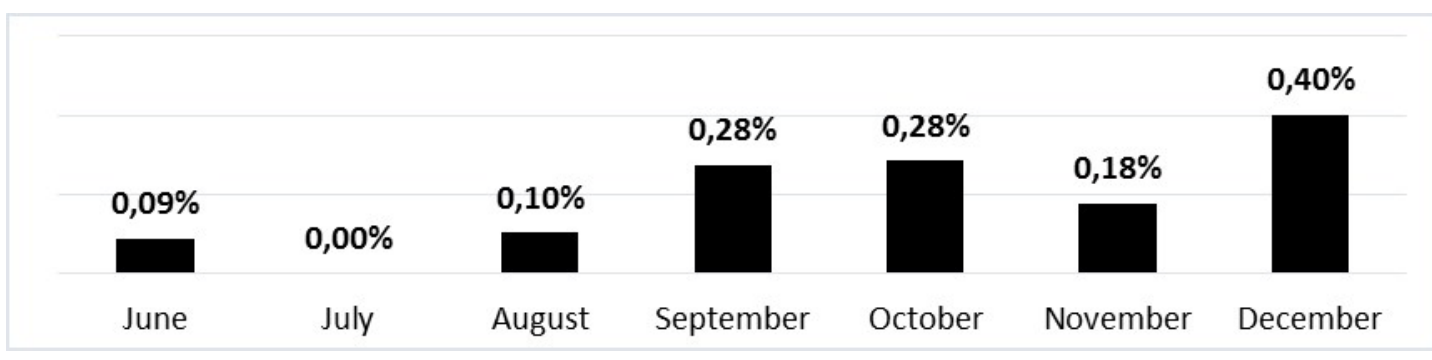

Figure 1. Isolation rate of NTM

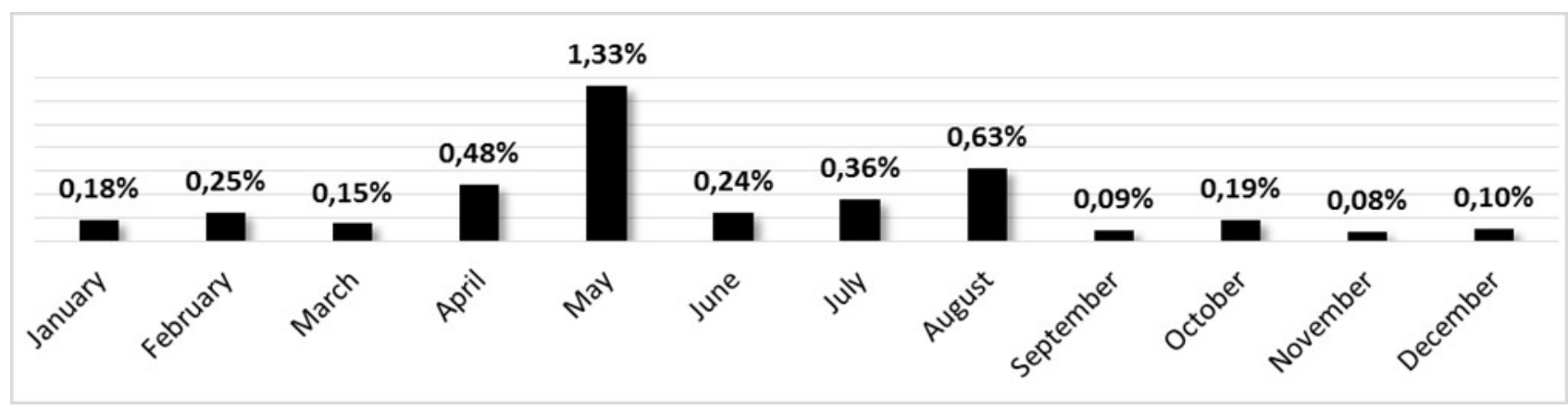

Figure 2. Isolation rate of NTM 
Table 1. NTM primary isolation on media

\begin{tabular}{|c|c|c|c|c|}
\hline \multicolumn{4}{|c|}{ Lowenstein-Jensen Medium } & \multirow{2}{*}{ Liquid medium BBL-MGIT } \\
\hline 21 days & 30 days & 45 days & 60 days & \\
\hline 5 & 7 & 14 & 16 & 21 \\
\hline
\end{tabular}

Table 2. Age range for positive NTM patients

\begin{tabular}{cccccc}
\hline Patients age range & $<20$ & $20-40$ & $40-60$ & $60-80$ & $>80$ \\
\hline No. of cases & 0 & 5 & 18 & 30 & 10 \\
\hline
\end{tabular}

phase and specific to the MTC members (Toihir et al., 2011).

\section{Results and discussions}

For the considered period, the overall isolation rate of NTM out of the total samples recorded $(n=25271)$ in the laboratory for diagnostic was $0.25 \%$. There were $14(0.18 \%)$ NTM isolates in $2015,48(0.34 \%)$ in 2016 and only one in the first three months of 2017. The values match the findings mentioned in the literature (van der Werf et al., 2014). The monthly isolation rates vary from 0 to $1.33 \%$, the last in May 2016 (Figure 1 and 2).

The majority of the samples (approx. 97\%) were isolated from respiratory tract specimens, mainly sputum (70\%), only two being extrapulmonary samples, pus and tissue biopsy. No acid fast bacilli were observed at the microscopic examination. Twenty one samples registered growth only in the automated system, on liquid medium, BBL-MGIT, and 37 only on LJ solid medium, only 5 of the samples presented growth on both media (Table 1.).More often, the growth on solid media was observed after 45-60 days of incubation, $38 \%$ of the NTM isolates were registered positive after two months at $37^{\circ} \mathrm{C}$.

Approximately $83 \%$ of the samples originated from patients from Iasi County and the other 17\%, from neighboring regions: Bacău $(n=2)$, Vaslui $(\mathrm{n}=2)$ Neamț $(\mathrm{n}=4)$, Botoşani $(\mathrm{n}=1)$, Suceava $(n=2)$. Most of the records were new cases $(87 \%)$, 4 being registered in treatment monitoring and 4 as relapses.
The majority of the samples came from patients with age over 60 years old, $30 \%$ with age between 60 and 70 and the median age was 62.3years (Table 2). From female patients were isolated $43 \%$ of the NTM and $57 \%$ from males.

\section{Conclusions}

NTM infections are not systematically reported by public health authorities and therefore epidemiological and surveillance data are not available, despite their importance in the diagnosis and management of these conditions. In view of the increasing incidence of infections caused by these bacteria, recorded in many countries, there is a need for evaluation of the NTM species involved in both human and veterinary pathology. The clinical significance of NTM isolation from human specimens is to be carefully evaluated by the clinicians, given the difficult interpretation whether the isolated species are the etiology of the disease or just due tocolonization.

Acknowledgments. This research did not receive any specific grant from funding agencies in the public, commercial, or not-for-profit sectors.

\section{References:}

1. Griffith DE et al. (2007). An official ATS/IDSA statement: diagnosis, treatment, and prevention of nontuberculous mycobacterial diseases. American Journal of Respiratory and Critical Care Medicine, 175(4):367-416.

2. Hoefsloot W, van Ingen J, Andrejak C, Angeby K, Bauriaud $\mathrm{R}$, Bemer P, et al. (2013). The geographic diversity of Nontuberculous Mycobacteria isolated from pulmonary samples: A NTM-NET collaborative study. Eur Respir J, 42:1604-13. 
3. Johnson MM, Odell JA (2014). Nontuberculous mycobacterial pulmonary infections. J. Thorac. Dis., 6:210-220.

4. Panagiotou M, Papaioannou AI, Kostikas K, Paraskeua M, Velentza E, Kanellopoulou M, Filaditaki V, Karagiannidis N (2014). The epidemiology of pulmonary nontuberculous mycobacteria: data from a general hospital in Athens, Greece, 2007-2013. Pulm Med. 894976.

5. Qvist T, Gilljam M, Jönsson B, Taylor-Robinson D, JensenFangel S, Wang M, Svahn A, Kötz K, Hansson L, Hollsing A, Hansen CR, Finstad PL, Pressler ., Høiby N, Katzenstein TL, Scandinavian Cystic Fibrosis Study Consortium (SCFSC) (2015). Epidemiology of nontuberculous mycobacteria among patients with cystic fibrosis in Scandinavia. J Cyst Fibros, 14:46-52.
6. Rindi L, Garzelli C (2016). Increase in non-tuberculous mycobacteria isolated from humans in Tuscany, Italy, from 2004 to 2014. BMC Infectious Diseases, 16, article 44.

7. Toihir AH, Rasolofo V, Andrianarisoa SH, Ranjalahy GM, Ramarokoto H (2011). Validation of an immunochromatographic assay kit for the identification of the Mycobacterium tuberculosis complex. Mem Inst Oswaldo Cruz., 106(6):777-80.

8. Van der Werf MJ, Ködmön C, Katalinić-Janković V, Kummunik T, Soini H, Richter E, Papaventisis D, Tortoli E, Perrin M, van Soolingen D, Zoinir-Dovć M, Thomsen VØ (2014). Inventory Study of Nontuberculous Mycobacteria in European Union. BMC Infect Dis., 14:62. 